Pacific Journal of Mathematic 


\title{
REMARK ON THE PRECEDING PAPER OF CHARLES LOEWNER
}

\author{
G. SzEGÖ
}

1. Introduction. In the preceding paper, Charles Loewner constructed certain Jordan curves with the property that the clamped plates bounded by such Jordan curves have an oscillating Green's function. The question concerning the sign of the Green's function has been raised by J. Hadamard, and this problem has been pursued recently by R. J. Duffin and P. R. Garabedian. The construction of Loewner is based on a method due to N. Muskhelichvili using appropriate conformal mappings. ${ }^{1}$

The purpose of the present note is to construct such Jordan curves in an elementary manner. For the sake of completeness we repeat a few definitions to be found in the preceding paper.

A function of $u(x, y)$ defined in a domain $g$ and having therein continuous partial derivatives of the fourth order is called a biharmonic function in $g$ if it satisfies the biharmonic equation

$$
\nabla^{4} u=\nabla^{2} \nabla^{2} u=\frac{\partial^{4} u}{\partial x^{4}}+2 \frac{\partial^{4} u}{\partial x^{2} \partial y^{2}}+\frac{\partial^{4} u}{\partial y^{4}}=0 .
$$

Let $g$ be a connected domain bounded by a finite number of analytic arcs. Let $q$ be a fixed point in $g$. The Green's function $\Gamma(p)=\Gamma(p ; q)$ of $g$ with respect to $q$ is a function of the variable point $p=p(x, y)$ satisfying the following conditions:

(a) $\Gamma$ is a biharmonic function of $p$ except at the singular point $q$. Denoting by $r$ the distance of $p$ from $q$, we have

$$
\Gamma=r^{2} \log r+k,
$$

where $k(p)=k(x, y)$ is biharmonic in $g$ without exception.

(b) On the boundary of $g$ we have the conditions:

\footnotetext{
${ }^{1}$ See the References given in the paper of Loewner.
}

Received June 23, 1952.

Pacific J. Math. 3 (1953), 437-446 


$$
\Gamma=\frac{\partial \Gamma}{\partial n}=0 .
$$

A function $u(x, y)$ biharmonic in the neighborhood of $x=0, y=0$ can be written in the form:

$$
u(x, y)=\left(x^{2}+y^{2}\right) u_{1}(x, y)+u_{2}(x, y)
$$

where $u_{1}$ and $u_{2}$ are harmonic functions in the neighborhood of $x=y=0$.

The previous concepts can be extended to infinite domains by using an analogue of Thomson's transformation. As is obvious from the representation (4), we have:

Let $u(x, y)=u(r, \phi)$ be harmonic in the neighborhood of the origin $r=0$ ( $r, \phi$ are polar coordinates $)$. We apply the inversion

$$
\begin{array}{rlrl}
x=x^{\prime}\left(x^{\prime 2}+y^{\prime 2}\right)^{-1}, & & y=y^{\prime}\left(x^{\prime 2}+y^{\prime 2}\right)^{-1}, \\
r & =\left(r^{\prime}\right)^{-1}, & \phi & =\phi^{\prime} .
\end{array}
$$

The function

$$
U\left(x^{\prime}, y^{\prime}\right)=\left(r^{\prime}\right)^{2} u(x, y)
$$

will be then a biharmonic function in the neighborhood of $x^{\prime}=\infty, y^{\prime}=\infty$.

A function biharmonic in the neighborhood of the origin can be presented as a linear combination of the basic biharmonic functions

$$
\begin{array}{cl}
r^{n} \cos n \phi, & r^{n} \sin n \phi, \\
r^{n+2} \cos n \phi, & r^{n+2} \sin n \phi,
\end{array}
$$

to which $r^{2} \log r$ has to be added if the function is singular at the origin (as for instance is the case for the Green's function with respect to the origin).

A function biharmonic in the neighborhood of $x=\infty, y=\infty$, can be represented as a linear combination of the basic biharmonic functions

$$
\begin{array}{ccc}
r^{-n} \cos n \phi, & r^{-n} \sin n \phi, & \\
r^{2-n} \cos n \phi, & r^{2-n} \sin n \phi, & n=0,1,2, \cdots,
\end{array}
$$

to which $\log r$ has to be added if the function is singular at infinity.

By use of the inversion (5), (6) there is no difficulty in defining the Green's function of an infinite domain with singular point at infinity provided that this point is an interior point of the domain. 
2. Results. In order to prepare the construction announced above, we consider the infinite plane, which we interpret as the complex $z$-plane, $z=x+i y=$ $r e^{i \phi}$, cut along the following circular arc of the unity circle:

$$
r=1, \pi-\alpha \leqq \phi \leqq \pi+\alpha .
$$

Here $\alpha$ is given, $0<\alpha<\pi$. We map this infinite slit domain onto the exterior of the unit circle of the $\zeta$-plane, $\zeta=\rho e^{i \psi}$, in such a way that $z=\infty$ and $\zeta=\infty$ correspond to each other. Furthermore, we assume that $d z / d \zeta$ is positive at $z=\zeta=$ $\infty$. This mapping has the following form:

$$
z=\frac{\zeta(\lambda \zeta-1)}{\zeta-\lambda}, \quad 0<\lambda<1
$$

Here $\lambda$ is an appropriate function of $\alpha$.

First we note that the real point $z=-1$ of the slit corresponds to $\zeta=1$ and $\zeta=-1$. Now let $\lambda=\cos \psi_{0}, 0<\psi_{0}<\pi / 2$; since

$$
\frac{d z}{d \zeta}=\lambda \frac{1-2 \lambda \zeta+\zeta^{2}}{(\zeta-\lambda)^{2}}
$$

we see that the points $\zeta=e^{ \pm i \psi_{0}}$ correspond to the end-points $z=-e^{ \pm i \alpha}$ of the slit. More precisely, $e^{i \psi_{0}}$ corresponds to $e^{i(\pi-a)}=-e^{-i a}$. As $\zeta=e^{i \psi}$ describes the unit circle in the positive sense, $z$ surrounds the circular slit; the $\operatorname{arc}-\psi_{0}<$ $\psi<\psi_{0}$ corresponds to the inner ( concave) side of the slit, and the remaining arc to the outer ( convex) side of it. In particular, $\zeta=1$ and $\zeta=-1$ are transformed into the point $z=-1$ on the concave and convex border of the slit, respectively.

Inserting $\zeta=e^{i \psi_{0}}$ in (2), we find that

$$
\frac{e^{i \psi_{0}}\left(\cos \psi_{0} \cdot e^{i \psi_{0}}-1\right)}{e^{i \psi_{0}}-\cos \psi_{0}}=\frac{e^{2 i \psi_{0}}\left(\cos \psi_{0}-e^{-i \psi_{0}}\right)}{e^{i \psi_{0}}-\cos \psi_{0}}=e^{2 i \psi_{0}}=-e^{-i \alpha},
$$

so that $2 \psi_{0}=\pi-\alpha$; hence

$$
\lambda=\sin (\alpha / 2)
$$

We denote the image of the circle $|\zeta|=R, R>1$, by $C_{R}$. This is an analytic Jordan curve.

The principal results of this note are the following:

I. Let $\Gamma(p)$ be the Green's function of the infinite slit domain of the $z$-plane 
bounded by the circular arc (1), having at $z=\infty$ its singular point. This function changes its sign in the slit domain just defined.

II. Let $\Gamma(p)$ be the Green's function of the infinite domain outside of the curve $C_{R}, R>1$, having at $z=\infty$ its singular point. This function will change its sign in the infinite domain outside of $C_{R}$ provided $R$ is sufficiently near to 1 .

From the last example it is easy to derive an example of the kind announced in the introduction: we have to apply an inversion to the curve $C_{R}$ with respect to any fixed interior point. Here we must use the results of Chapter 1 .

3. Circular slit. We seek the Green's function $\Gamma(p)$ of the circular slit domain in the following form:

$$
\Gamma(p)=\log \frac{1}{\rho}+A\left(\rho-\frac{1}{\rho}\right) \cos \psi+f(\rho, \psi)+\left(r^{2}-1\right) g(\rho, \psi) .
$$

Here $A$ is a constant, and $f(\rho, \psi)$ and $g(\rho, \psi)$ are harmonic functions regular for $\rho>1$, including $\rho=\infty$. The point $p$ is represented by the complex number $z=r e^{i \phi}$ defined in 2. The relation between $z=r e^{i \phi}$ and $\zeta=\rho e^{i \psi}$ is given by $2(2)$.

The boundary conditions of the clamped plate amount to the fact that the function (1) and its derivative with respect to $\rho$ vanish as $\rho=1$. But $\rho=1$ implies that $r=1$, so that we have:

$$
\begin{aligned}
& \text { (I) } f(1, \psi)=0 \text {; i.e., } f(\rho, \psi) \equiv 0 . \\
& \text { (II) }-1+2 A \cos \psi+\left(\frac{\partial\left(r^{2}\right)}{\partial \rho}\right)_{\rho=1} g(1, \psi)=0 .
\end{aligned}
$$

Now we note the following formulas which will be useful in our later work:

$$
\begin{aligned}
r^{2} & =\rho^{2} \frac{\lambda^{2} \rho^{2}-2 \lambda \rho \cos \psi+1}{\rho^{2}-2 \lambda \rho \cos \psi+\lambda^{2}}, \\
r^{2}-1 & =\left(\rho^{2}-1\right) \frac{-2 \lambda \rho \cos \psi+\lambda^{2}\left(\rho^{2}+1\right)}{\rho^{2}-2 \lambda \rho \cos \psi+\lambda^{2}} .
\end{aligned}
$$

From the second we conclude that

$$
\left(\frac{\partial\left(r^{2}\right)}{\partial \rho}\right)_{\rho=1}=2\left(\frac{-2 \lambda \rho \cos \psi+\lambda^{2}\left(\rho^{2}+1\right)}{\rho^{2}-2 \lambda \rho \cos \psi+\lambda^{2}}\right)_{\rho=1}=\frac{4 \lambda(-\cos \psi+\lambda)}{1-2 \lambda \cos \psi+\lambda^{2}} .
$$


Hence condition (II) can be written as follows:

$$
-1+2 A \cos \psi+\frac{4 \lambda(-\cos \psi+\lambda)}{1-2 \lambda \cos \psi+\lambda^{2}} g(1, \psi)=0 .
$$

We determine $A$ according to the condition

$$
\begin{gathered}
-1+2 A \cos \psi+\frac{4 \lambda(-\cos \psi+\lambda)}{4 \lambda^{2}}=0, \\
A=(2 \lambda)^{-1},
\end{gathered}
$$

and (4) yields

( 5$)$

$$
g(1, \psi)=\frac{1-2 \lambda \cos \psi+\lambda^{2}}{4 \lambda^{2}} ;
$$

$$
g(\rho, \psi)=\frac{1+\lambda^{2}}{4 \lambda^{2}}-\frac{1}{2 \lambda} \frac{\cos \psi}{\rho} .
$$

Recapitulating, we find the following expression for the Green's function $\Gamma(p)$ :

(6) $\Gamma(p)=\log \frac{1}{\rho}+\frac{1}{2 \lambda}\left(\rho-\frac{1}{\rho}\right) \cos \psi+\left(r^{2}-1\right)\left(\frac{1+\lambda^{2}}{4 \lambda^{2}}-\frac{\cos \psi}{2 \lambda \rho}\right)$.

In the limiting case $\alpha \longrightarrow \pi, \lambda \rightarrow 1$, we obtain of course the Green's function of the exterior of the unit circle, namely,

$$
\log \frac{1}{\rho}+\frac{1}{2}\left(\rho^{2}-1\right) .
$$

4. Conclusion. (a) The dominant term in $3(6)$ is

$$
r^{2} \frac{1+\lambda^{2}}{4 \lambda^{2}}
$$

so that $\Gamma$ is positive as $z \longrightarrow \infty$. Now we write

$$
z=r=0, \zeta=\rho=\frac{1}{\lambda},
$$

and have 
(1)

$$
\begin{aligned}
\Gamma & =\log \lambda+\frac{1}{2 \lambda}\left(\frac{1}{\lambda}-\lambda\right)-\left(\frac{1+\lambda^{2}}{4 \lambda^{2}}-\frac{1}{2}\right) \\
& =\log \lambda+\frac{1-\lambda^{2}}{4 \lambda^{2}} .
\end{aligned}
$$

This quantity is certainly negative if $\lambda$ is sufficiently near 1 , more precisely if $\lambda>\lambda_{0}$ where $\lambda_{0}$ is the only root of the equation

$$
\log \lambda+\frac{1-\lambda^{2}}{4 \lambda^{2}}=0
$$

on the range $0<\lambda<1$.

(b) We can show however that $\Gamma$ must change its sign for all $\lambda, 0<\lambda<1$. For this purpose we compute the following second derivative at the point $z=-1$ on the concave side of the slit:

$$
\begin{aligned}
\left(\frac{d^{2} \Gamma}{d \rho^{2}}\right)_{\rho=1, \psi=0}=1-\frac{1}{\lambda} & +\left(\frac{d^{2}\left(r^{2}\right)}{d \rho^{2}}\right)_{\rho=1, \psi=0} \frac{(1-\lambda)^{2}}{4 \lambda^{2}} \\
& +\left(\frac{d\left(r^{2}\right)}{d \rho}\right)_{\rho=1, \psi=0} \cdot \frac{1}{\lambda} .
\end{aligned}
$$

From the second formula in $3(2)$ we find

$$
\begin{aligned}
& \left(\frac{d^{2}\left(r^{2}\right)}{d \rho^{2}}\right)_{\rho=1, \psi=0}=2 \frac{-2 \lambda+2 \lambda^{2}}{(1-\lambda)^{2}} \\
& +4 \frac{\left(-2 \lambda+2 \lambda^{2}\right)(1-\lambda)^{2}-\left(-2 \lambda+2 \lambda^{2}\right)(2-2 \lambda)}{(1-\lambda)^{4}}=\frac{4 \lambda(1+3 \lambda)}{(1-\lambda)^{2}},
\end{aligned}
$$

so that, in view of $3(3)$,

$$
\left(\frac{d^{2} \Gamma}{d \rho^{2}}\right)_{\rho=1, \psi=0}=1-\frac{1}{\lambda}+\frac{1+3 \lambda}{\lambda}-\frac{4}{1-\lambda}=-\frac{4 \lambda}{1-\lambda}
$$

which is indeed negative.

(c) It is interesting to compute this second derivative for all values of $\lambda$. We obtain from $3(6)$ : 


$$
\begin{aligned}
\left(\frac{\partial^{2} \Gamma}{\partial p^{2}}\right)_{\rho=1} & =1-\frac{\cos \psi}{\lambda}+2 \frac{-2 \lambda \cos \psi+2 \lambda^{2}}{1-2 \lambda \cos \psi+\lambda^{2}}\left(\frac{1+\lambda^{2}}{4 \lambda^{2}}-\frac{\cos \psi}{2 \lambda}\right) \\
& +4 \frac{d}{d \rho}\left(\frac{-2 \lambda \rho \cos \psi+\lambda^{2}\left(\rho^{2}+1\right)}{\rho^{2}-2 \lambda \rho \cos \psi+\lambda^{2}}\right)_{\rho=1}\left(\frac{1+\lambda^{2}}{4 \lambda^{2}}-\frac{\cos \psi}{2 \lambda}\right) \\
& +\frac{2 \cos \psi}{\lambda} \frac{-2 \lambda \cos \psi+2 \lambda^{2}}{1-2 \lambda \cos \psi+\lambda^{2}} \\
& =\frac{4 \lambda(\lambda-\cos \psi)}{1-2 \lambda \cos \psi+\lambda^{2}} .
\end{aligned}
$$

Hence this second derivative is positive on the convex side of the circular arc, and negative on the concave side of this arc. On the convex side $\Gamma$ is positive, and on the concave side $\Gamma$ is negative, provided $p$ is sufficiently near to the arc in question.

\section{On the Green's function of the infinite domain which is the exterior of $C_{R}$.} We denote now by $\Gamma^{\prime}(p)$ the Green's function of the infinite domain which is the exterior of $C_{R}$, having its singular point at infinity. We seek this function in the form:

$$
\begin{aligned}
\Gamma(p)=\log \frac{R}{\rho} & +A \frac{\rho}{R} \cos \psi+B \\
& +C \frac{\rho^{2}-\lambda^{2}}{\rho^{2}-2 \lambda \rho \cos \psi+\lambda^{2}}+|z|^{2}\left(D+E \frac{R}{\rho} \cos \psi\right),
\end{aligned}
$$

where $A, B, C, D, E$ are appropriate constants depending on $R$ and $\lambda$; here again the point $p$ is represented by the complex number $z=r e^{i \phi}$, and the relation between $z=r e^{i \phi}$ and $\zeta=\rho e^{i \psi}$ is the same as above. We show that the constants $A, \cdots, E$ can be determined in a unique way so that $\Gamma$ satisfies the boundary conditions of the clamped plate provided that $R$ is sufficiently near to 1 ; more precisely, there must be $0<R-1<\epsilon=\epsilon(\lambda)$.

The conditions

$$
\Gamma=\frac{\partial \Gamma}{\partial \rho}=0
$$$$
\text { for } \rho=R
$$

are equival ent to the following: 


$$
\Gamma_{1}=\frac{\partial \Gamma_{1}}{\partial \rho}=0
$$

where the function $\Gamma_{1}$ is defined by

$$
\begin{aligned}
\Gamma_{1}= & \left(\rho^{2}-2 \lambda \rho \cos \psi+\lambda^{2}\right) \Gamma \\
= & \left(\log \frac{R}{\rho}+A \frac{\rho}{R} \cos \psi+B\right)\left(\rho^{2}-2 \lambda \rho \cos \psi+\lambda^{2}\right)+C\left(\rho^{2}-\lambda^{2}\right) \\
& +\rho^{2}\left(1-2 \lambda \rho \cos \psi+\lambda^{2} \rho^{2}\right)\left(D+E \frac{R}{\rho} \cos \psi\right) .
\end{aligned}
$$

Here we used the first formula in $3(2)$. Now (4) is a quadratic expression in $\cos \psi$. Conditions ( 3 ) can be replaced by the corresponding set of equations for the coefficients of $\cos \psi$ in $(4)$. These equations are somewhat simplified if in (4) we replace $\cos \psi$ by $\rho^{-1} \cos \psi$. The resulting coefficients are:

$$
M_{1}(\rho)=\left(\log \frac{k}{\rho}+B\right)\left(\rho^{2}+\lambda^{2}\right)+C\left(\rho^{2}-\lambda^{2}\right)+D \rho^{2}\left(1+\lambda^{2} \rho^{2}\right),
$$

(5) $M_{2}(\rho)=\frac{A}{R}\left(\rho^{2}+\lambda^{2}\right)-2 \lambda\left(\log \frac{R}{\rho}+B\right)-2 \lambda D \rho^{2}+E R\left(1+\lambda^{2} \rho^{2}\right)$, $M_{3}(\rho)=-\frac{\dot{2} \lambda A}{R}-2 \lambda E R$.

The boundary conditions are equivalent to the following set of conditions:

$$
M_{1}(R)=M_{1}^{\prime}(R)=M_{2}(R)=M_{2}^{\prime}(R)=M_{3}(R)=M_{3}^{\prime}(R)=0 .
$$

(b) The last of these six equations can be disregarded since $M_{3}(\rho)$ is independent of $\rho$. The resulting five equations are linear in the five unknown quantities $A, \cdots, E$. They are as follows:

$$
\begin{aligned}
& B\left(R^{2}+\lambda^{2}\right)+C\left(R^{2}-\lambda^{2}\right)+D R^{2}\left(1+\lambda^{2} R^{2}\right)=0, \\
& 2 R B-\frac{1}{R}\left(R^{2}+\lambda^{2}\right)+2 C R+D\left(2 R+4 \lambda^{2} R^{3}\right)=0, \\
& \frac{A}{R}\left(R^{2}+\lambda^{2}\right)-2 \lambda B-2 \lambda D R^{2}+E R\left(1+\lambda^{2} R^{2}\right)=0, \\
& 2 A+\frac{2 \lambda}{R}-4 \lambda D R+2 E \lambda^{2} R^{2}=0, \quad \frac{A}{R}+E R=0 .
\end{aligned}
$$


In order to show that the unknowns are uniquely determined, we have to discuss the determinant of this system. As $R \rightarrow 1$ the elements of this determinant approach those of the following determinant (the second and fourth equations are divided by 2 ):

$$
\left|\begin{array}{ccccc}
0 & 1+\lambda^{2} & 1-\lambda^{2} & 1+\lambda^{2} & 0 \\
0 & 1 & 1 & 1+2 \lambda^{2} & 0 \\
1+\lambda^{2} & -2 \lambda & 0 & -2 \lambda & 1+\lambda^{2} \\
1 & 0 & 0 & -2 \lambda & \lambda^{2} \\
1 & 0 & 0 & 0 & 1
\end{array}\right| .
$$

Subtracting here the first column from the last we obtain:

$$
\begin{aligned}
& \left(1-\lambda^{2}\right)\left|\begin{array}{cccc}
0 & 1+\lambda^{2} & 1-\lambda^{2} & 1+\lambda^{2} \\
0 & 1 & 1 & 1+2 \lambda^{2} \\
1+\lambda^{2} & -2 \lambda & 0 & -2 \lambda \\
1 & 0 & 0 & 0
\end{array}\right| \\
& =-\left(1-\lambda^{2}\right)\left|\begin{array}{ccc}
1+\lambda^{2} & 1-\lambda^{2} & 1+\lambda^{2} \\
1 & 1 & 1+2 \lambda^{2} \\
-2 \lambda & 0 & -2 \lambda
\end{array}\right| \\
& =2 \lambda^{2}\left(1-\lambda^{2}\right) \\
& \left|\begin{array}{cc}
1+\lambda^{2} & 1-\lambda^{2} \\
-2 \lambda & 0
\end{array}\right|=4 \lambda^{3}\left(1-\lambda^{2}\right) \neq 0 .
\end{aligned}
$$

6. Conclusion. From $5(7)$ it is obvious that the parameters $A, \ldots, E$ are rational functions of $R$ and $\lambda$. Let $\lambda$ be fixed, $0<\lambda<1$. Then these parameters are rational functions of $R$, and the evaluation of the determinant 5 (8) shows that they are regular in a certain neighborhood of $R=1$. Incidentally, we find from $3(6)$ that

$$
A=-E=\frac{1}{2 \lambda}, \quad D=-B=\frac{1+\lambda^{2}}{4 \lambda^{2}}, \quad C=0 \quad \text { as } R=1
$$

Inserting $z=0, \rho=1 / \lambda, \psi=0$ in 5 (1), we obtain an elementary function of $R$ which is regular at $R=1$. It is a combination of $\log R$ and the rational functions $A, B, C$ of $R$. Now this function is negative for $R=1$ (provided $\lambda$ is sufficiently small). From this the same property follows for the function 5 ( 1 ) provided $R$ is sufficiently near to 1 . This yields the desired property of the 
domain outside of the level curve $C_{R}$ of the conformal mapping of the circular slit domain onto the exterior of the unit circle.

In order to prove the same property for all $\lambda$ (and for sufficiently small values of $R-1$ ), we compute

$$
\left(\frac{d^{2} \Gamma}{d \rho^{2}}\right)_{\rho=R, \psi=0} .
$$

We note that the curve $C_{R}$ intersects the real axis in two points; the curve is convex at the left point and concave at the right point of the intersection, provided $R-1$ is small enough. The second derivative (2) we consider is associated with the concave point of intersection. Now (2) has the same sign as

$$
\left(\frac{d^{2} \Gamma_{1}}{d \rho^{2}}\right)_{\rho=R, \psi=0},
$$

where $\Gamma_{1}$ is defined as in $5(4)$. From 5 (4) we see again that $(3)$ is a function of $R$ which is regular at $R=1$. Since it is negative for $R=1$, it must be negative for all $R>1$ sufficiently near to 1 .

This establishes the assertions.

STANFORd UNIVERSITY 


\section{PACIFIC JOURNAL OF MATHEMATICS}

\section{EDITORS}

\section{R. U. FOEINSON}

University of California

Berkeley 4, California

E. HewitT

University of Washington

Seattle 5 , Washington
P. P. DILWOR TH

California Institute of Technology

Pasadena 4, California

E. F. BECKENBACH

University of California

Los Angeles 24, California

\section{ASSOCIATE EDITORS}

$\begin{array}{llll}\text { H. BUSEMANN } & \text { P. R. HALMOS } & \text { BØRGE JESSEN } & \text { J. J. STOKER } \\ \text { HERBERT FFDERER } & \text { IIEINZ HOPF } & \text { PAUL LÉVY } & \text { E. G. STRAUS } \\ \text { MARSHALL, IIALI } & \text { R. D. JAMES } & \text { GEORGE PÓLYA } & \text { KÖSAKU YOSIDA }\end{array}$

\section{SPONSORS}

UNIVERSITY OF BRITISH COLUMBIA

CALIFORNIA INSTITUTE OF TECHNOLOGY.

UNIVERSITY OF CAIJIFORNIA, BERKELEY

UNIVERSITY OF CAIJIFORNIA, DAVIS

UNIVERSITY OF CALIFORNIA, LOS ANGELES

UNIVERSITY OF CALIFORNI A, SANTA BARBARA

UNIVERSITY OF NEVADA

OREGON STATE COLLEGE

UNIVERSITY OF OREGON

\author{
UNIVERSITY OF SOU TIERN CALIFORNIA \\ STANFORD RESEARCH INSTITUTE \\ STANFORD UNIVERSITY \\ WASHINGTON STATE COLLEGE \\ UNIVERSITY OF WASHINGTON \\ AMERICAN MATHEMATICAL SOCIETY \\ NATIONAL BUREAU OF STANDARDS, \\ INSTITUTE FOR NUMERICAL ANALYSIS
}

Mathematical papers intended for publication in the Pacific Journal of Mathematics should be typewritten (double spaced), and the author should keep a complete copy. Manuscripts may be sent to any of the editors except Robinson, whose term expires with the completion of the present volume; they might also be sent to M.M. Schiffer, Stanford University, Stanford, California, who is succeeding Robinson. All other communications to the editors should be addressed to the managing editor, E. F. Beckenbach, at the address given above.

Authors are entitled to receive 100 free reprints of their published papers and may obtain additional copies at cost.

The Pacific Journal of Mathematics is published quarterly, in March, June, September, and December. The price per volume (4 numbers) is $\$ 8.00$; single issues, $\$ 2.50$. Special price to individual faculty members of supporting institutions and to individual members of the American Mathematical Society: $\$ 4.00$ per volume; single issues, $\$ 1.25$.

Subscriptions, orders for back numbers, and changes of address should be sent to the publishers, University of California Press, Berkeley 4, California.

Printed at Ann Arbor, Michigan. Entered as second class matter at the Post Office, Berkeley, California.

\section{UNIVERSITY OF CALIFORNIA PRESS • BERKELEY AND LOS ANGELES}




\section{Pacific Journal of Mathematics}

\section{Vol. 3, No. 2 \\ April, 1953}

William George Bade, An operational calculus for operators with spectrum

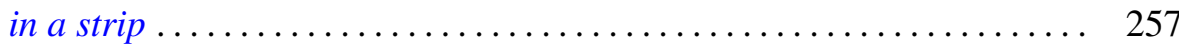

E. F. Beckenbach and Lloyd Kenneth Jackson, Subfunctions of several variables ..................................... 291

David Blackwell, Extension of a renewal theorem ................. 315

L. Carlitz, Some theorems on the Schur derivative ................ 321

Paul Arnold Clement, Generalized convexity and surfaces of negative curvature..................................... 333

Merrill M. Flood, On the Hitchcock distribution problem ............... 369

Watson Bryan Fulks, On the unique determination of solutions of the heat

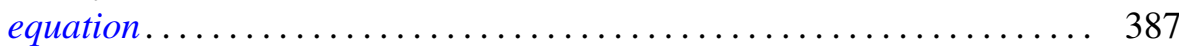

John W. Green, Length and area of a convex curve under affine transformation .................................... 393

William Gustin, An isoperimetric minimax .................. 403

Arthur Eugene Livingston, Some Hausdorff means which exhibit the Gibbs'

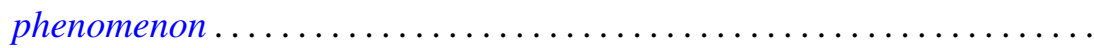

Charles Loewner, On generation of solutions of the biharmonic equation in the plane by conformal mappings ..................... 417

Gábor Szegő, Remark on the preceding paper of Charles Loewner ....... 437

Imanuel Marx and G. Piranian, Lipschitz functions of continuous

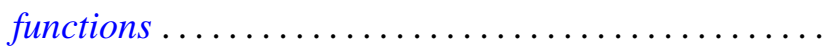

Ting-Kwan Pan, The spherical curvature of a hypersurface in Euclidean space ..

Ruth Lind Potter, On self-adjoint differential equations of second order ...

E. H. Rothe, A note on the Banach spaces of Calkin and Morrey...

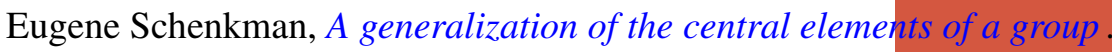

A. Seidenberg, A note on the dimension theory of rings .. . . 